\title{
A Geometrical Interpretation of the Symmetrical Invariant of Three Ternary Quadratics
}

\author{
Bу T. Sсотт, Emmanuel College, Cambridge. \\ (Received 2nd December, 1935. Read 6th December, 1935.)

\section{Introduction.}

In the paper, "Sul sistema di tre forme ternarie quadratiche," Ciamberlini ${ }^{1}$ has derived the complete irreducible system of concomitants for three ternary quadratics and has given a short treatment of their geometrical interpretations. Among the concomitants is the invariant $(a b c)^{2}$ which is symmetrical and linear in the coefficients of each quadratic. The purpose of this note is to give a geometrical interpretation of the invariant, and to extend the result for symmetrical invariants of forms in higher dimensions. ${ }^{2}$

\section{$\S 1$. Notation.}

In symbolic form the point and line equations of the three conics are taken to be:-

$$
\begin{array}{ll}
f_{1}=a_{x}^{2}=a_{x}^{\prime 2}=\ldots, & \phi_{1}=u_{a}^{2}=u_{a^{\prime}}^{2}=\ldots \\
f_{2}=b_{x}^{2}=b_{x}^{\prime 2}=\ldots, & \phi_{2}=u_{\beta}^{2}=u_{\beta^{\prime}}^{2}=\ldots \\
f_{3}=c_{x}^{2}=c_{x}^{\prime 2}=\ldots, & \phi_{3}=u_{\gamma}^{2}=u_{\gamma^{\prime}}^{2}=\ldots
\end{array}
$$

where $\quad a_{x}=\sum_{i=1}^{3} a_{i} x_{i}, \quad u_{a}=\sum_{i=1}^{3} u_{i} a_{i}, \quad a=\left(a a^{\prime}\right)$.

The equation in symbolic form of the $\Phi$-conic for $f_{1}, f_{2}$ is easily obtained from binary forms by the use of the Clebsch Transference Principle.

The invariant $(a b)^{2}=0$ signifies that the pairs of points represented by the binary equations $a_{x}^{2}=0, b_{x}^{2}=0$ form a harmonic range on a line, and so extending to ternary forms we have

$$
(a b u)^{2}=0
$$

as the envelope of lines cutting the conics $f_{1}, f_{2}$ in harmonic point pairs-i.e. the $\Phi$-conic for $f_{1}, f_{2}$.

1 Giorn. di Mat., Napoli 24 (1886), 141.

2 My thanks are due to Professor Turnbull who has superintended the work and given me much valuable advice and assistance. 
Dually, we obtain $(\alpha \beta x)^{2}=0$ as the equation of the $F$-conic.

We shall use the notation

$$
\Phi_{23}=(b c u)^{2}, \quad \Phi_{31}=(c a u)^{2}, \quad \Phi_{12}=(a b u)^{2},
$$

to denote the three $\Phi$-conics associated with $f_{1}, f_{2}, f_{3} ; \Phi_{i j}$ denoting the harmonic envelope of $f_{i}, f_{j}$.

From these results it is obvious that

$$
(a b c)^{2}=0
$$

is the condition for the conic locus $f_{i}$ to be outpolar to the conic envelope $\Phi_{j k}$, where $i, j, k=1,2,3$ and $i, j, k$ are all different.

This, in fact, is the interpretation ascribed to $(a b c)^{2}$ by Ciamberlini. The following pages, however, set out a piece of geometry more in keeping with the symmetrical nature of the invariant.

\section{§2. The invariant $(a b c)^{2}$.}

It will now be proved that, if $u$ is any line in the plane and $u_{i}$ the polar with respect to $f_{i}$ of the pole of $u$ with respect to $\Phi_{j k}$, then $u_{1}, u_{2}, u_{3}$ are concurrent when $(a b c)^{2}=0$.

Likewise, if we take a point $P$ in the plane, then the three points $P_{1}, P_{2}, P_{3}$ are collinear, where $P_{i}$ is the pole with respect to $\Phi_{j k}$ of the polar of $P$ with respect to $f_{i}$.

Consider a line $u$. Then its pole with respect to $\Phi_{12}$ is

$$
(a b u)(a b v)=0, \quad(v, \text { current coordinates }),
$$

and the polar of this point with respect to $f_{3}$ is

$$
\left(u_{1}\right), \quad(a b u)(a b c) c_{x}=0, \quad(x, \text { current). }
$$

Thus, we get the three connexes

$\left(u_{1}\right),(a b c)(b c u) a_{x}=0, \quad\left(u_{2}\right),(a b c)(c a u) b_{x}=0, \quad\left(u_{3}\right),(a b c)(a b u) c_{x}=0$, defining the three lines $u_{1}, u_{2}, u_{3}$ associated with the fixed line $u$. By the fundamental identity for determinantal permutations we have

$$
(a b c)(b c u) a_{x}+(a b c)(c a u) b_{x}+(a b c)(a b u) c_{x} \equiv(a b c)^{2} u_{x}
$$

Hence, if

$$
(a b c)^{2}=0 \text {, }
$$

the three lines $u_{1}, u_{2}, u_{3}$ are concurrent in a point $P$. We may, however, start with a fixed point $P$ and obtain three points $P_{1}, P_{2}, P_{3}$ collinear on $u$.

We obtain a dual interpretation in terms of the $F$-conics for $(\alpha \beta \gamma)^{2}=0$ by using the identity

$$
(\alpha \beta \gamma)(\beta \gamma x) u_{\alpha}+(\alpha \beta \gamma)(\gamma \alpha x) u_{\beta}+(\alpha \beta \gamma)(\alpha \beta x) u_{y} \equiv(\alpha \beta \gamma)^{2} u_{x} .
$$




\section{§3. Extension to Three Dimensions.}

For four quaternary quadratic forms

$$
f_{1}=a_{x}^{2}, \quad f_{2}=b_{x}^{2}, \quad f_{3}=c_{x}^{2}, \quad f_{4}=d_{x}^{2},
$$

$(a b c u)^{2}=0$ represents the quadric envelope of planes cutting the quadrics $f_{1}, f_{2}, f_{3}$ in three conics having the symmetrical property considered above.

This follows by application of the Clebsch Transference Principle to the ternary invariant $(a b c)^{2}$.

Hence, we obtain four quadric envelopes which we can specify by

$$
\Phi_{123}=(a b c u)^{2}, \quad \Phi_{234}=(b c d u)^{2}, \quad \Phi_{341}=(c d a u)^{2}, \quad \Phi_{412}=(d a b u)^{2} .
$$

As in $\S 2$, the vanishing of $(a b c d)^{2}$, the symmetrical invariant of four quadrics, is the condition for any $f_{i}$ to be outpolar with respect to $\Phi_{j k l}$, where $i, j, k, l=1,2,3,4$, and $i, j, k, l$ are all different.

If, however, we consider the four connexes

$$
\begin{array}{llll}
\left(u_{1}\right), & a_{x}(a b c d)(b c d u)=0, & \left(u_{2}\right), & b_{x}(a b c d)(c d a u)=0, \\
\left(u_{3}\right), & c_{x}(a b c d)(d a b u)=0, & \left(u_{4}\right), & d_{x}(a b c d)(a b c u)=0,
\end{array}
$$

then, since

$(a b c d)(b c d u) a_{x}+(a b c d)(c a d u) b_{x}+(a b c d)(a b d u) c_{x}+(a b c d)(a c b u) d_{x} \equiv(a b c d)^{2} u_{x}$ it follows that $(a b c d)^{2}=0$ is the condition for the four planes $u_{1}, u_{2}$, $u_{3}, u_{4}$ to meet in a point, where $u_{i}$ is the polar with respect to $f_{i}$ of the pole of $u$ with respect to $\Phi_{j k l}$.

Dually, we can interpret $(\alpha \beta \gamma \delta)^{2}=0$ by using the quadric loci $(\alpha \beta \gamma x)^{2}=0$ etc. It is obvious that by repeated application of the Clebsch Transference Principle it is possible to interpret $(a b c . p)^{2}=0$ for $p$ quadrics in $[p-1]$.

§4. $(a b c)^{2}$ in relation to the F-conics of the $\Phi$-conics.

In this paragraph we suppose $(a b c)^{2}=0$. The $F$-conic of $\Phi_{12}$, $\Phi_{23}$, is

$$
F_{(12)(23)} \equiv\left(a b \cdot b^{\prime} c \cdot x\right)^{2}=0 .
$$

Thus

$$
\left[\left(\dot{a} b^{\prime} c\right) \dot{b}_{x}\right]^{2}=0 \text {, }
$$

and

$$
(a b c)^{2} f_{2}+c_{\beta}^{2} f_{1}-2\left(a b^{\prime} c\right) b_{x}\left(b b^{\prime} c\right) a_{x}=0
$$

so that

$$
(a b c)^{2} f_{2}+{ }_{x} a_{\beta} c_{x}=0
$$

and ${ }_{x} a_{\beta} c_{x}=0$ represents the conic which is the locus of a point $x$ whose polars with respect to $f_{1}, f_{3}$ are conjugate lines with respect to $\phi_{2}$. 
From the result (1) we see that the conic ${ }_{x} a_{\beta} c_{x}=0$ meets $f_{2}$ in four points which are vertices of harmonic pencils of tangents to $\Phi_{12}, \Phi_{23}$. For, since $(a b c)^{2}=0$, it follows that

$$
\begin{array}{ll} 
& F_{(12)(23)} \equiv{ }_{x} a_{\beta} c_{x}=0, \\
& F_{(23)(31)} \equiv_{x} a_{\gamma} b_{x}=0, \\
\text { and } \quad F_{(31)(12)} \equiv_{{ }} b_{a} c_{x}=0 .
\end{array}
$$

The condition for $d_{x}^{2} \equiv{ }_{x} a_{\beta} c_{x}$ to be outpolar with respect to $\Phi_{31}$ is $(c a d)^{2}=0$,

i.e.,

$$
\left(c a a^{\prime}\right) a_{\beta}^{\prime} c_{\beta}^{\prime}\left(c a c^{\prime}\right)=0 .
$$

Hence

$$
\frac{1}{2} c_{\alpha}^{2} c_{\beta}^{2}-\frac{1}{2} c_{\alpha} c_{\alpha}^{\prime} c_{\beta} c_{\beta}^{\prime}=0 \text {, }
$$

and therefore $(\alpha \beta \gamma)^{2}=\mathbf{0}$.

Thus, for $(a b c)^{2}=0$ and $(\alpha \beta \gamma)^{2}=0$ simultaneously, the symmetrical property holds for the $\Phi$-conics and their $F$-conics. 